\title{
A MULTI-AGENT METHOD FOR ADAPTIVE REAL-TIME TRAIN SCHEDULING WITH CONFLICT LIMITATIONS
}

\author{
A.A. BELOUSOV ${ }^{1,2}$, A.A. GORYACHEV ${ }^{2}$, P.O. SKOBELEV 3 \& M.E. STEPANOV ${ }^{1,2}$ \\ ${ }^{1}$ Samara State Aerospace University (National Research University), Russia. \\ ${ }^{2}$ SEC "Smart Solutions", Russia. \\ ${ }^{3}$ Group of companies "Knowledge Genesis", Russia.
}

\begin{abstract}
The paper proposes a multi-agent method to creating an intelligent adaptive system of train real time scheduling with conflict limitations. The architecture of the multi-agent system consisting of two base planning subsystems is described. Subsystem interaction protocols and protocols of agent interaction within each subsystem are presented. The example of schedule planning in various situations is presented in details. Realizable characteristics of the developed multiagent system are presented. High quality of schedule planning and system performance is shown.

Keywords: conflict limitations, conjugate interactions method, intelligent systems, multiagent systems, real time planning, swarm systems.
\end{abstract}

\section{INTRODUCTION}

The modern development of railway transport is characterized by a constant increase of passenger traffic and extent of trailing cargos; new high-speed trains appear, time intervals between trains become shorter, train-handling capacity of the railway network reaches limit value. Due to the high traffic intensity, trains are highly interconnected: changes in one train's schedule, or a conflict with this train, will affect the next train and can have impact on the whole train network in a very unpredictable way. In this case, rescheduling of all trains in the operating domain might be required, which should be done quickly, in real-time, and this is a very difficult task, given the whole variety of planning conditions, preferences and limitations as well as security requirements.

In spite of a high level of railway network automation today, solving conflict situations in trains deviating from the master-plan completely depends on the experience of the dispatcher in charge, which often leads to irrational placing of trains in the traffic, especially in stressful situations. The constantly growing scale of the task to be solved brings up the increasing complexity of disruptive situations and provokes a question as how to reduce dependency on the human factor by automating the decision-making process and introducing intelligent systems, enabling fast and effective adjustments in case of a disruptive event.

An intelligent system should make decision 'on the fly' under condition of constantly changing context of the situation, which requires correct changes to be made on time and the previously developed train schedule to be adjusted. Thus, to solve this task one has to reject the assumption of the equilibrium of the environment. Modern intelligent systems designed to help in real-time decisionmaking in complex and large-scale systems should use new methods and means of automation management, enabling to take into account the whole variety of factors, conditions, rules, and interactions, considering that limitations apply individually and can be regulated in the process of work, provide high level of precision and quality of decisions as well as productivity.

This paper describes the multi-agent approach, which solves the complex task of adaptive realtime train management. At present, the developed system is adapted in production use on a section of the high-speed rail between Saint Petersburg and Moscow and between Saint Petersburg and Buslovskaya. 


\section{MULTI-AGENT APPROACH TO ADAPTIVE TRAIN ROUTE MANAGEMENT}

\subsection{Problem statement}

The task of adaptive real-time train route management consists of creating a detailed train route plan with minimal deviations from the master-plan, considering various limitations and requirements for continuously incoming events (trains in the infrastructure block sections, train damage, track occupancy, maintenance, speed limitations, etc.).

The solution of this task is the optimal plan of placing trains delayed due to unforeseen events back into the schedule in real time, as well as list of dispatcher regulation measures for solving conflict situations and implementing the plan.

The input data consists of: railway infrastructure with detailed block sections (stations, railway switches, block sections), requirements for train schedule (master-plan), maintenance requirements, updates on the current situation in the operating domain about trains and state of block sections (signals of busy condition, information on unavailability). It is worth mentioning that the scale of the task is enormous which makes it a large-scale task.

The main limitations of the system are traffic security requirements, normative route-building requirements, train priorities, dispatcher rules, etc.

However, besides the limitations listed above, there are ones that are hard to formalize (no unjustified changing of tracks, no traffic jams between stations, no unjustified halts or trains stays on the main tracks, correct routing of trains by dispatcher schedule, etc.), which should be taken into consideration while planning. At the same time, the implementation of any kind of requirements depends on the current situation, that is, there is situational decision-making.

For instance, a train may not choose wrong track, but if there is a busy block section on its path or a maintenance window, then it can do so, in order to bypass the obstacle and stay on time - provided its maneuvering does not affect other trains with higher priorities. On the other hand, it may stay on the same track and wait for a while, if this delay is not big and the train can catch up. But waiting at a station means stopping for a little while, and a train may only choose block sections of certain length for stay and so on. Hence, a decision whether the route should be changed or not is connected to many conditions that require finding balance and consensus.

For solving the task of adaptive real-time train route management, a system based on multi-agent methods and technologies has been developed. Multi-agent approach allows considering the whole varieties of conditions, limitations and requirements for train management, including ones that are hard to formalize, by having dialog between agents and finding consensus satisfying all sides of conflict. Architecture of the multi-agent system is open and enables introduction new conditions and criteria as well as changing ones already existing and managing them dynamically.

The multi-agent system for solving the task of adaptive real-time train route management runs on Vektor-M program platform [1,2], which allows for keeping the dynamic infrastructure model of the operating domain, get signals from block sections, appointed maintenance windows, satellite and other information.

\subsection{Architecture of the multi-agent system}

The architecture of the developed system is built on network-centric principles, where every subsystem has its own individual task and the final solution is reached through negotiating between individual decisions [3]. 
The primary plan building takes place in two subsystems. Each subsystem builds a train route schedule on its own level of understanding of the scene in such a way that the initial rough decision is transformed into a more precise one. A decision made in every subsystem is conflict-free for its level of understanding (no converging train routes, the security requirements are intact). This layerbased train scheduling eliminates the combinatorial explosion of possibilities, make the scheduling process more stable to disruptions due to a reduction in the scale of the task at higher levels and step-by-step consideration of all possible limitations according to the level of importance and impact on other layers.

All events arriving in the system can be divided into two main types: new request or update on the current situation. Requests in their turn can be of two basic types: request to let the train pass on schedule or request to conduct maintenance works. Update on the current situations can be either a train moving along block sections or a state of an infrastructure (damage or busy condition).

The first level of planning is represented by a trajectory scheduler, the second one by a time scheduler. A general decision-making method and the role of schedulers in it are depicted in Fig. 1.

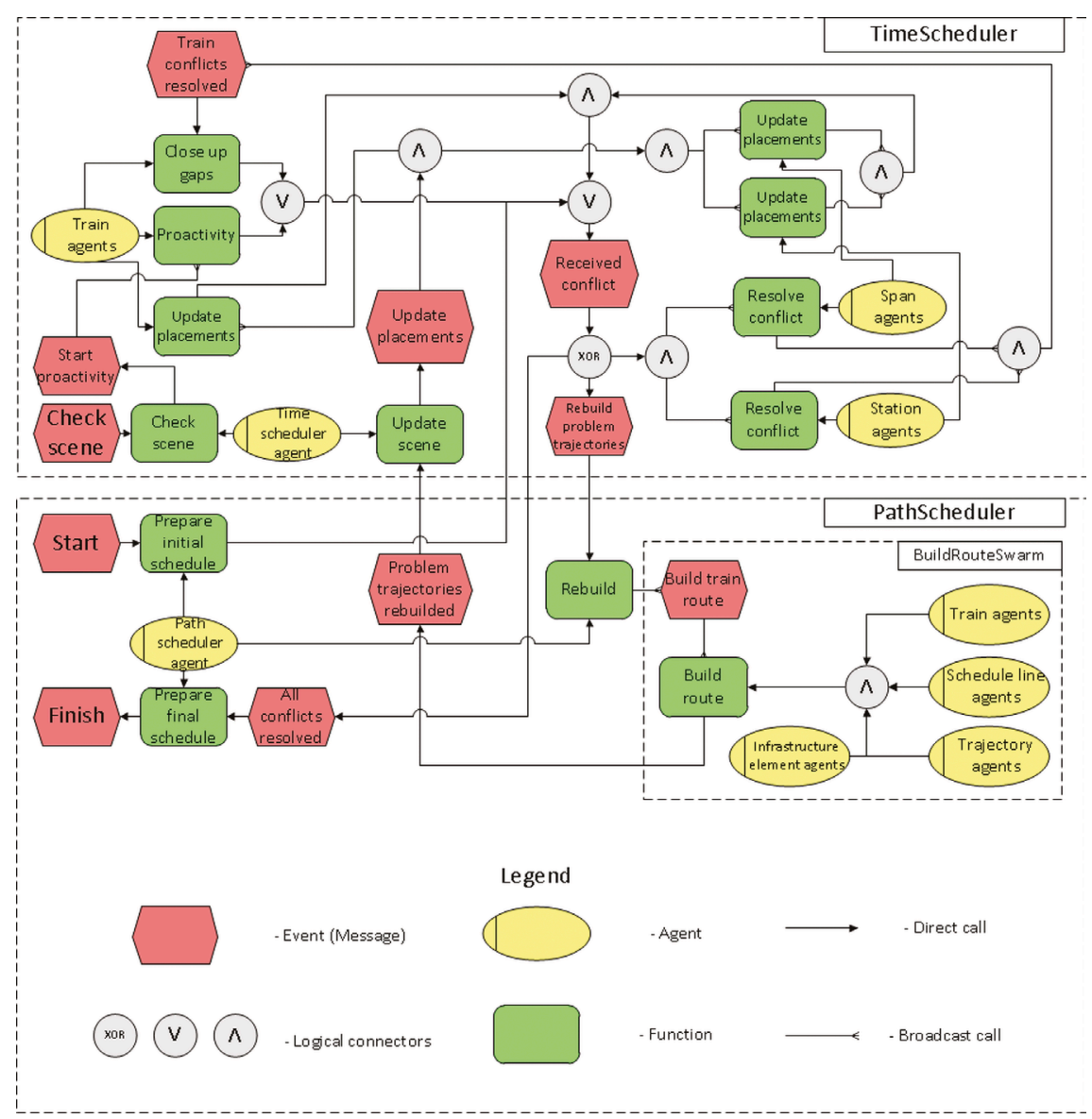

Figure 1: Interaction between main planning subsystems, represented by a diagram event-driven process chain. 
The diagram describes interaction between systems on the high level of abstraction. The diagram blocks in their turn can be subsystems with complex inner structure.

Work in the system starts with a message 'Start', which is sent to the 'Path scheduler agent'. In response, the function 'Prepare initial schedule' appears, which prepares the planning scene, after which the event 'Received conflict' appears in the time scheduler. In the result, the time scheduler detects current schedule conflicts and determines further direction. If no conflicts are found, 'Path scheduler agent' is sent a message 'All conflicts resolved', and by processing the message the function 'Prepare final schedule' forms the overall schedule. If conflicts are found, there are two variants of events: to send the message 'Rebuild problem trajectories' to the trajectory scheduler or to solve the conflict on its level by changing time of resources occupation and sending 'Resolve conflict' to station and station limits agents.

In the first case, the trajectory scheduler will change tracks and planned block sections of trains' stops in the schedule problem zone and will send a message 'Problem trajectories are rebuilt' to 'Time scheduler agent', then the agent 'Update scene' is called, which starts link updating process of requirement- possibilities network between requirements of train traffic and infrastructure elements, after which the event 'Received conflict' emerges again in the time scheduler.

In the second case, in the process of resolving conflicts in their own schedule, station and station limits agents create conflicts in train schedule, so called 'Gap', which appear due to the difference in arrival and departure time on the adjacent elements of infrastructure. Solving 'Gap' conflicts takes place in the method 'Close up gap', implemented by train agents, resulting in new conflicts of resources occupancy, which is checked when event 'Received conflict' appears. Reaching a compromise between train agents and agents of infrastructure elements (stations and station limits) is the final objective of the planning system.

If a compromise is reached and all conflicts are resolved, but decision does not satisfy the requirements, a message 'Check scene' initializes the process of proactivity, as a result of which certain agents try to improve their own schedule and cause a new field of resolving conflicts.

Time scheduler represents an operating domain as a set of station limits and stops, which builds a train schedule in less detail, conflicts are resolved by queuing the trains, speeding them up and slowing them down.

Time planning is similar to a visual schedule analyzing. The main task is to build a new possible train schedule considering the normative schedule limitations and train priorities. The solution is based on the method of conjugate interactions for managing resource allocation in real time [4]. On this level, a train agent creates subtasks (operations) for passing a station limit or staying at a station for a certain amount of time. An agent of every subtask of this kind looks for a placement for itself in the respective resource, trying to find the most profitable position by negotiating with other subtask agents. High-priority trains are more active in finding a placement (have more energy for pushing other requests for resources).

The main decision-making condition here is accomplishing the task with minimal divergence:

$$
D E V_{T}=\sum_{i=1}^{N}\left(\left|T D_{S}-T P_{F}\right|+\left|T D_{F}-T P_{F}\right|\right) \rightarrow \min
$$

where TDs - scheduled starting time, TDf - scheduled finishing time, TPs - actual starting time, TPf - actual finishing time, $\mathrm{N}$ - number of resources (station limits, station platforms), where operations of passing and stopping can be implemented. Additional conditions implemented while making decisions are listed below. The result of the time scheduler system's work is a schedule for stay and 
passing stations and station limits in an operating domain, which is sent to the trajectory component in order to build train routes according to block sections, provided it is possible.

While scheduling on the time scheduler, there are two types of interactions:

1. interaction between interval agents of different trains on one block section: this algorithm enables us to provide for an order change of moving trains on block sections;

2. interaction between interval agents of one train on different block sections.

This algorithm enables to keep the trajectory entirety of each train (if a train is late at some block section, it requires correcting the schedule of this train on all its block sections)

Each type of interaction is used for the decision making of respective type of conflict, and one type of conflict might result in appearance of conflicts of another type.

In the trajectory subsystem, a trajectory on block sections is built according to the calculated graphic in less detail; conflicts are resolved by bypassing and route changing.

The primary task of scheduling paths and stays is to allocate routes for trains to take and choose the block section for their stays, considering overlapping routes of arriving and leaving for the parking. In this subsystem, a train agent creates new subtask agents that look for routes for passing the station and station limit according to the condition of minimal route costs. 'Cost' is the cumulative key performance indicator of the route, which includes different normative requirements for train routes (correct or not, length, number of connections). After scheduling with minimal KPIs, station route agents enter the active phase of lifecycle, where the main condition for decision-making is no overlapping of train routes in a block section. When such an overlap is found, a station route agent will try to transmit one of the conflicting subtasks to other route agents. Route agents communicate via the task exchange protocol $[5,6]$.

Building and negotiating the final train schedule takes place in a close interaction between scheduler levels. In every planning subsystem, there is a swarm of agents representing the levels between the subsystems; there are back links that come into play when a conflict cannot be resolved locally in the current subsystem. The primary allocation of tasks to resources is done based on the best decision possible independently and in concurrent threads, which allows for reduction of computing time by excluding the rest of possibilities. Such 'greedy' allocation results in conflicts that are resolved by agents grouping together into structures within a swarm - domains. In each domain, searching for a compromise takes place between agents in order to resolve the conflict.

\subsection{Criteria for schedule quality assessment}

The setting of optimization criteria is based on several requirements imposed on the final schedule. These requirements are collected from system users, and the main ones are listed below. For convenience, all criteria are grouped.

Group 'Initial planning'

1) No unjustified deviations from master-plan. Deviations can be justified by:

- Inconsistency between schedule and infrastructure (no available stay at station, no 4.5-minute train spacing, forced passing loop on a single-track road, noncompliance of bulk cargo graphics, etc.).

- Impact of disruptive situations (windows, train delay/advance, busy condition, speeding down or train priority, etc.). 


\section{Group 'Conflicting movement organization'}

2) No unjustified choosing the wrong (conflicting) track. Choosing the wrong (conflicting) track can be justified by:

- non-bypassing window on the right track or bypassing window(s), which can cause delay of more than 4 minutes (breaking/acceleration average time);

- fixing the train on a wrong track or block sections, from which only entrance to the wring track is possible.

\section{Group 'Stays planning'}

3) No unjustified train stays. Justified stays are:

- stays justified by master-plan;

- stays caused by a passing loop on a single-track line;

- stays caused by trains with lower priorities giving way to ones with high priority;

- stays caused by bypassing of obstacles, if there is no possibility to pass the obstacle;

- 24-hour stays caused by speeding down of trains.

4) No train stays on tracks with not enough length.

5) No stays of heavy-loaded trains on 'heavy profile track'.

6) No long stays on main tracks (for instance, suburban trains stay 1 minute).

7) Sustaining the given intervals of non-simultaneous train arrivals to a station.

Group 'Routing of dispatcher schedule trains'

8) Routing of dispatcher schedule trains should not lead to deviations of other trains, except for trains with the same priority as dispatcher schedule train.

9) While routing of dispatcher schedule trains, there should be no unjustified stays. An example of a justified stay is giving way to passenger trains. Theoretically, arrival should pass the operating domain without stays at stations.

\section{SOFTWARE IMPLEMENTATION}

\subsection{Realization features}

For performance analysis of the multi-agent system for adaptive real-time train route management, the following time indices were used: infrastructure load time, train load time in an operating domain, rescheduling time for a new arrival, rescheduling time depending on duration of maintenance window, rescheduling time for added maintenance windows, rescheduling time depending on the number of tracks with maintenance windows, rescheduling time depending on speed limits on a block section, rescheduling time depending on the number of limitations, speed on a block section, rescheduling time depending on the number of tracks occupied due to a speed limit at a station.

A train schedule in operating domain has around 45 operations (operations of passing, stay at a station or passing a station), every train operation has its own agent. There are around 800 trains in total, so total number of agents is around 36,000. Apart from that, there are train agents - around 800 , station agents -49 , station route agents -500 , block section agents -3700 , maintenance request and availability agents - around 100-200. Dividing this many agents into levels and grouping them 
into isolated swarms of agents that are active at certain points in time allows increasing the performance [3].

To get realization features for each index, average value was determined based on the results of 10 experiments for two operating domains: Saint Petersburg-Buslovskaya and Saint PetersburgMoscow.

Figures relevant for planning the features of the operating domains are represented in Table 1.

The Moscow-Saint Petersburg operating domain has 2.8 times more infrastructure objects than the Saint Petersburg-Buslovskaya operating domain. The load time of the Moscow-Saint Petersburg operating domain is about 4.9 times higher than on the other operating domain. It is caused by building of the infrastructure model within the system and special infrastructure station agents, station limits, block sections.

Table 2 represents the change of planning scheduling characteristics depending on the number of scheduled trains on the operating domains.

According to Table 2, the difference between the scheduling times on the operating domains with around the same number of trains is 2.6 times, which is close to the difference between domain infrastructures. Thus, dependency can be observed, which is close to linear, between the number of infrastructure elements and the scheduling time of trains on an operating domain.

Rescheduling time is $15-40 \%$ less in comparison with initial scheduling time. It is caused by adaptive rescheduling based on incoming events instead of rescheduling everything from scratch. Increasing the number of trains increases the train scheduling time in direct proportion.

Table 1: Relevant figures of operating domains.

\begin{tabular}{lcccc}
\hline Operating domain & $\begin{array}{c}\text { Number of } \\
\text { stations }\end{array}$ & $\begin{array}{c}\text { Number of } \\
\text { infrastructure } \\
\text { objects }\end{array}$ & $\begin{array}{c}\text { Number of } \\
\text { turnouts }\end{array}$ & $\begin{array}{c}\text { Average load and } \\
\text { preparation time of } \\
\text { infrastructure (ms) }\end{array}$ \\
\hline Saint Petersburg - Buslovskaya & 17 & 1293 & 133 & 1452 \\
Saint Petersburg - Moscow & 47 & 3640 & 304 & 7194 \\
\hline
\end{tabular}

Table 2: Planning scheduling characteristics depending on the number of tasks.

\begin{tabular}{lccc}
\hline Operating domain & $\begin{array}{c}\text { Number of } \\
\text { trains }\end{array}$ & $\begin{array}{c}\text { Average scheduling time } \\
(\mathrm{ms})\end{array}$ & $\begin{array}{c}\text { Average rescheduling time } \\
(\mathrm{ms})\end{array}$ \\
\hline Saint Petersburg - & 12 & 1511 & 1185 \\
Buslovskaya & 17 & 2180 & 1429 \\
& 30 & 2257 & 1799 \\
& 49 & 2300 & 2092 \\
& 67 & 2746 & 2356 \\
Saint Petersburg - & 71 & 7202 & 5063 \\
Moscow & 86 & 8786 & 6164 \\
& 152 & 12691 & 11420 \\
& 241 & 22453 & 22899 \\
& 311 & 36143 & 37351 \\
\hline
\end{tabular}


Table 3 represents planning scheduling characteristics depending on the density of disruptions. Increasing the density increases the scheduling time.

The scheduling time mostly depends on the number of infrastructure elements and the number of scheduled trains. Other factors such as disruptions, their number, duration and density have less influence on the total scheduling time. This can be explained by partial (adaptive) scheduling according to incoming disruptive events instead of complete rescheduling. Thus, one can speak about guaranteed time for decision making in given infrastructure and given number of trains.

The following qualitative characteristics can be noted: no violations of given train arrival intervals, no unjustified changing of tracks, no traffic jams between stations, keeping security intervals, almost no delays among intercity and high-speed trains in conflict situations, average train delays less than 9\% (20 trains are in one conflict). This outcome has been achieved on such large-scale planning tasks for the first time.

\subsection{Example of resolved conflict situations}

Let us consider an example of how the system deals with conflict situations and take a closer look at the decisions made by the scheduler. The situation with a high number of disruptions is shown in Fig. 2. There are six maintenance windows, two of which completely block the traffic between two stations for an hour.

Resolving this situation required involving back links between different planning levels. Due to a high number of thickened graphic lines after the window, the trajectory subsystem was unable to create the final schedule, since the traffic security requirements did not allow the trains to stop and switch on to the alternative route. The trajectory subsystem registers this mismatch as a conflict and sends a message to the time subsystem. In order to resolve the situation, the time subsystem must delay a few trains from previous stations (for example, \#2048, \#6155, \#6163), taking the overload of the station limits into account. It sends the newly made decision to the trajectory subsystem, which builds the route and checks the decision to satisfy traffic security requirements. As a result, the schedule has turned out to be more balanced and stable to possible further disruptions, the effect of the maintenance window has been localized, after which the schedules tend to be exemplary again.

Table 3: Planning scheduling characteristics depending on the density of disruptions.

\begin{tabular}{llc}
\hline \multirow{2}{*}{ Operating domain } & \multicolumn{1}{c}{$\begin{array}{c}\text { Density of disruptions } \\
\text { Saint Petersburg - Buslovskaya }\end{array}$} & $\begin{array}{c}\text { Average scheduling time } \\
(\mathrm{ms})\end{array}$ \\
& No disruptions & 2356 \\
& Very few disruptions & 2476 \\
& Few disruptions of the same type & 2505 \\
& Many disruptions of different types & 2785 \\
Saint Petersburg - Moscow & No disruptions & 37351 \\
& Very few disruptions & 39631 \\
& Few disruptions of the same type & 37613 \\
& Many disruptions of different types & 39501 \\
\hline
\end{tabular}




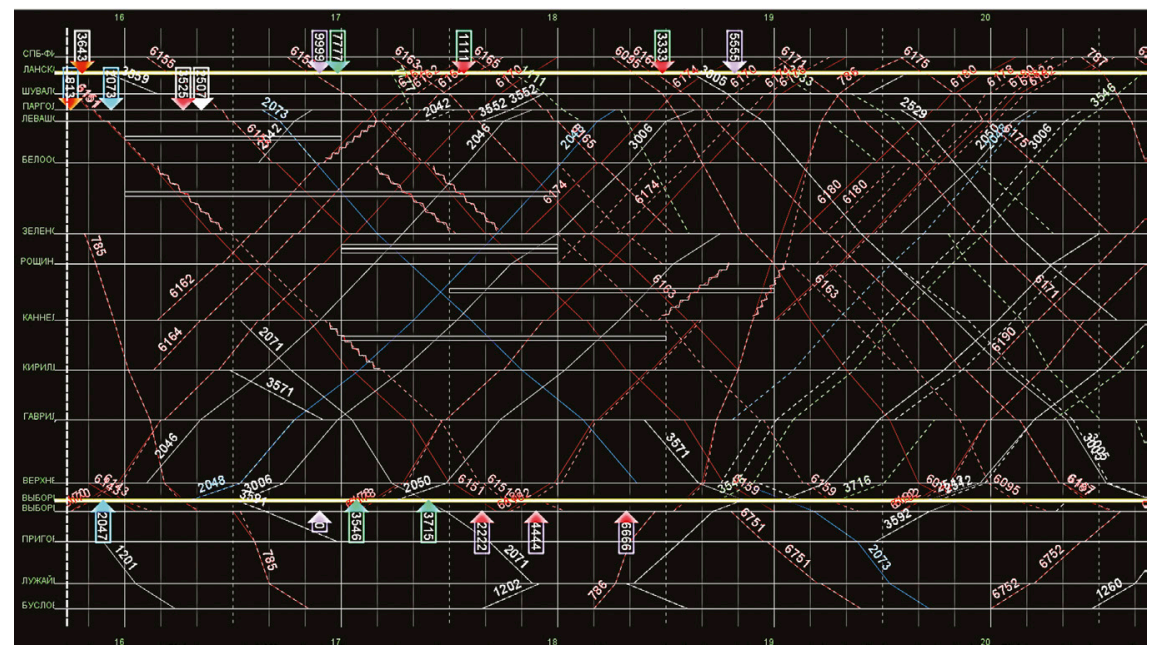

Figure 2. Bypassing six maintenance windows, resuming after the disruptions have been eliminated.

An overload between two stations cannot be resolved without the time subsystem, because changing the route with switching on to the opposite tracks will cost much more than changing the train schedule.

\subsection{Adoption indicators}

The expected results of the developed adaptive train scheduling system include: reduced reaction time, increased flexibility and quickness of decision-making in response to disruptive events, increased effectiveness of railway resource management in real time and securing on-time performance of trains, reduced man-hours for rescheduling trains, a completely new intelligent software system for traffic management in real time.

At present, there is no detailed statistics about indicators of planning the system in comparison with dispatcher work. However, one can already be sure about reduced dispatcher reaction time on unpredictable events, reduced hours for routing train traffic with dispatcher schedule, increased quality of final schedule and correspondence to the strict requirements.

For instance, train routing with dispatcher schedule represents a complex task, considering the whole variety of affecting factors (dimensions, weight, length in carriages, type of load, normative movement, speeding up and down time, etc.). It is preferable for this train to pass an operating domain as quickly as possible and with minimal number of stays, but at the same time not conflict with other trains. Complexity of dispatcher routing of a cargo train with bulk load is caused by interdiction of crossing with high-speed rail trains because of security requirements. But an experienced dispatcher can easily handle this task even with dense schedule. However, it will take him two or more minutes.

Routing of several trains from different directions under conditions of technologic works in a station limit requires correct calculation of all standards for speeding up and down while setting on a stay, choosing alternative tracks, all speed limitations, all track occupancy at a station, all lengths of free stays and so on. One should also remember customary trains that pass an operating domain and 
different unpredictable events happening. In this situation, dispatcher needs more time for decision making, at least a couple of minutes for each train.

In fact, schedule does not remain static, and due to external events might become impossible in several seconds. For example, busy condition on a track of required length at a station due to damage makes planned train stay impossible. Deviations from the master-plan spoil dispatcher's statistics, and hence affect his or her wages, so this situation results in greater attention of the dispatcher and can lower priority to the problem of qualitative train routing.

In case of delay of a high-priority train, it should be put back in the master-plan as quickly as possible. This is a critical and higher priority task in comparison with others. A dispatcher, acting according to the situation and taking into account low priority of bulk train, can put it on a timely stay, which one can avoid. At the same time, every unplanned stay of a cargo train requires money costs and has a negative effect on the dispatcher's statistics.

Hence, a dispatcher has to control a number of indicators and at the same time take into account his wages, but thoroughly thinking over low-priority tasks is not always possible. In turn, a scheduler, in spite of the variety of events, disruptions, conditions and limitations can manage the train routing task, including dispatcher schedule, in seconds and give the dispatcher a decision that satisfies all limitations and requirements, with minimal deviations from master-plan and minimal number of stays (reduces energy expenditures).

\section{CONCLUSION}

The suggested multi-agent system of adaptive real time train scheduling [3] has been developed within the project of the unified intelligent train scheduling system for the Russian railways $[7,8]$.

The following further developments of the scheduler are: explaining the decisions made, visualization of the decision space, demonstrating the decision logic to the dispatcher, interaction with the dispatcher for improving the quality of the schedule, modelling the future developments, teaching, evaluating the quality of the decision based on a flexible set of conditions and the common 'satisfaction' level of resources, increasing performance by paralleling asynchronous planning processes.

The project has been supported by the Ministry of Education and Science of Russian Federation in the framework of the implementation of the Program of increasing the competitiveness of SSAU among the world's leading scientific and educational centers for the years 2013-2020.

\section{REFERENCES}

[1] Shabunin, A.B., Markov, S.N., Dmitriev, D.V., Kuznetsov, N.A., Skobelev, P.O., Kozhevnikov, S.S., Simonova, E.B. \& Tsarev, A.V., Integrative platform for the network-centric approach to creating allocated intelligent resource management systems at Russian Railways. Software engineering, (9), pp. 23-28, 2012.

[2] Shabunin, A.B., Kuznetsov, N.A., Skobelev, P.O., Babanin, I.O., Kozevnikov, S.S., Simonova, E.V., Stepanov, M.E. \& Tsarev, A.V., Developing an ontology for the multi-agent resource management system for Russian railways, Information technologies. (12), pp. 42-45, 2012.

[3] Belousov, A.A., Skobelev, P.O., Efremov, G.A., Stepanov, M.E., Goryachev, A.A. \& Shabunin, A.B., Multi-agent approach to a complex task of train scheduling in a large-scale railway management systems, Papers of the MLSD '2014, Moscow, pp. 252-263, ISBN 978-5-91450$161-4$.

[4] Vittich V.A. \& Skobelev P.O. Method of conjugate interactions in allocated resource management in real time. Autometria, (2), pp. 78-87. 
[5] Abbink, E.J.W., Mobach, D.G.A., Fioole, P.J., Kroon, L.G., van der Heijden, E.H.T. \& Wijngaards, N.J.E., Actor-agent application for train driver rescheduling, Proceedings of AAMAS, Budapest, Hungary, pp. 513-520, 2009.

[6] Skobelev, P.O., Belousov, A.A., Lisicin, S.O. \& Tcarev, A.V., Development of the intelligent cargo logistics management system for Vostochny polygon, Papers of XV World Conference Problems of Management and Simulation in Complex Systems, Samara, 25-28 June 2013, Samara Science Centre RAS: Samara, pp. 391-396, 2013, ISBN 978-5-93424-662-5.

[7] Shabunin, A.B., Kuznetsov, N.A., Skobelev, P.O., Babanin, I.O., Kozevnikov, S.S., Simonova, E.V., Stepanov, M.E. \& Tsarev, A.V., Developing a multi-agent resource management system for Russian railways, Mechatronics, automation, management, (1), pp. 23-29, 2013.

[8] Matuchin V.G. \& Shabunin A.B. \& IRMS, Concept and implementation, Papers from the first conference Intelligent Railway Management Systems IRMS-2012, Moscow, 15-16 November 2012, pp. 15-18. 